\title{
Type-II stepwise progressive censoring
}

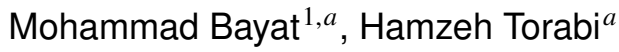 \\ ${ }^{a}$ Department of Statistics, Yazd University, Iran
}

\begin{abstract}
Type-II progressive censoring is one of the censoring methods frequently used in clinical studies, reliability trials, quality control of products and industrial experiments. Sometimes in Type-II progressive censoring experiments, the failure rate is low so the waiting time to observe the $m^{\text {th }}$ failure will be very long; however, the experimenter may have to terminate the experiment before a predetermined time. In this article, if two generalized types of Type-II progressive censoring are reminded, we then make some changes in the removal method of Type-II progressive censoring such that without reducing the deduction quality, the termination time of the experiment decreases. This can be done with decreasing withdraws throughout the steps of the experiment with a special reasonable method. A simulation study is done and the results are tabulated at the end of this article for a comparison between introduced method and Type-II progressive censoring.
\end{abstract}

Keywords: Type-II stepwise progressive censoring, Type-II progressive censoring, maximum likelihood estimator, lifetime experiment, failure rate, test duration

\section{Notation}

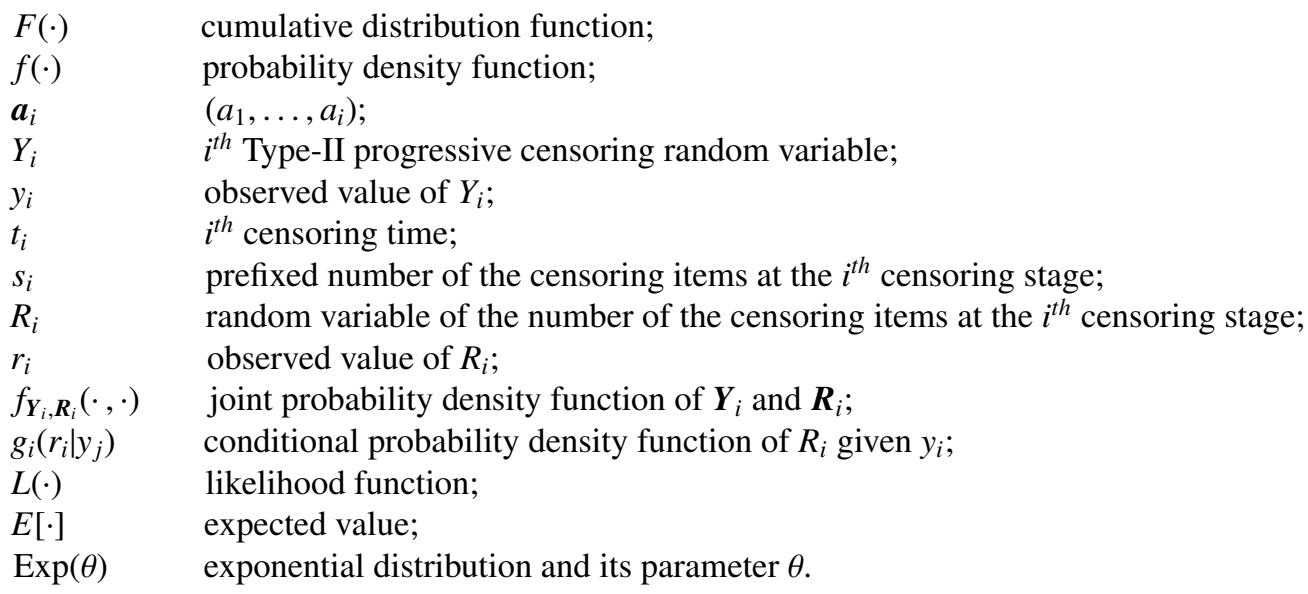

\section{Introduction}

Suppose that $n$ independent units with identical cumulative distribution function $(\mathrm{CDF} ; F(\cdot))$ and probability density function $(\mathrm{PDF} ; f(\cdot))$ are placed on a lifetime experiment. Moreover, assume that at the first failure time $\left(y_{1}\right), s_{1}$ surviving experimental units are removed and at the second failure time

\footnotetext{
${ }^{1}$ Corresponding author: Department of Statistics, Yazd University, 89175-741, Yazd, Irna. E-mail: bayat.stat@chmail.ir

Published 31 January 2016 / journal homepage: http://csam.or.kr

(c) 2016 The Korean Statistical Society, and Korean International Statistical Society. All rights reserved.
} 


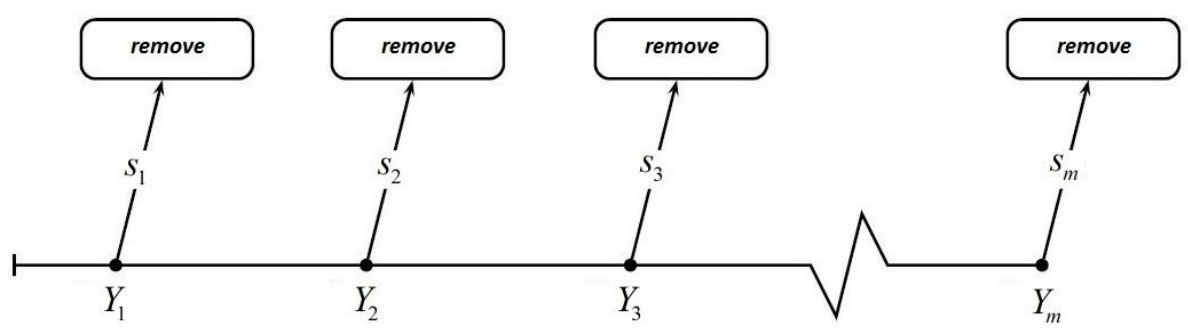

Figure 1: Type-II progressive censoring.

$\left(y_{2}\right), s_{2}$ surviving units are withdrawn, and so on. Finally, at the $m^{\text {th }}$ failure time $\left(y_{m}\right)$, all remaining surviving units $\left(s_{m}\right)$ are removed from the experiment; This censoring method is called "Type-II progressive censoring" and the observed values are $y_{1}, y_{2}, \ldots, y_{m}$. In this method, the number of observed failures, $m$, and the censoring removal scheme $s_{1}, s_{2}, \ldots, s_{m}$ are prefixed (Figure 1).

For more detail about Type-II progressive censoring readers can refer to Balakrishnan and Aggarwala (2000), Balakrishnan (2007), Balakrishnan and Cramer (2014), Balakrishnan et al. (2008, 2014), Burkschat (2008), Burkschat et al. (2006), Cohen (1963), Cramer (2014), Cramer and Kamps (2001), Herd (1956). Some valuable results can also be found in Ghitany et al. (2013, 2014), Kang and Seo (2011), Krishna and Kumar (2013), Pakyari and Balakrishnan (2013), Rezapour et al. (2013a, 2013b), Seo and Kang (2014). Cramer and Iliopoulos (2009) proposed an adaptive Type-II progressive censoring such that the number of removals has been involved in previous failure times and the number of previous censored items. Sarhan and Al-Ruzaizaa (2010), Dey and Dey (2014), and Tse et al. (2000) use the Binomial distribution as the CDF of the number of the removal items. Moreover, $\mathrm{Ng}$ et al. (2009) have generalized Type-II progressive censoring while making some modifications.

\subsection{Ng et al.'s method}

In $\mathrm{Ng}$ et al.'s method, similar to Type-II progressive censoring the censoring, removal scheme and a threshold time such as $T$ should be determined before the experiment. The experiment begins with $n$ independent and identically distributed units. At the first failure time $\left(y_{1}\right), s_{1}$ surviving units are randomly selected and removed if $y_{1}<T$ and otherwise no action is done. The experiment continues with all remaining items. Similarly, at the $j^{\text {th }}$ failure time, $2 \leq j \leq m-1, s_{j}$ units of surviving experimental units are removed from the experiment when $y_{j}<T$, else the experiment is continued without any removal. Finally, at the $m^{\text {th }}$ failure time, all remaining items are removed from the experiment and the experiment is terminated. Let removals at $j^{\text {th }}$ stage be denoted by $r_{j}, j=1, \ldots, m$. Therefore

$$
R_{j}= \begin{cases}s_{j}, & y_{j} \leq T \\ 0, & \text { other wise. }\end{cases}
$$

Figure 2 clarifies the method. In the figure, two main cases are shown ( $\mathrm{Ng}$ et al., 2009).

\subsection{Kinaci's method}

An extension of Type-II progressive censoring has been introduced by Bairamov and Parsi (2011). The extension of Bairamov's scheme has been given by Kinaci (2013). The Kinaci's method can be explained as follow. 


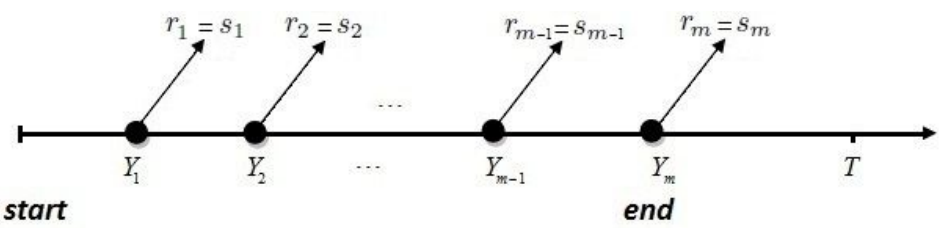

(a)

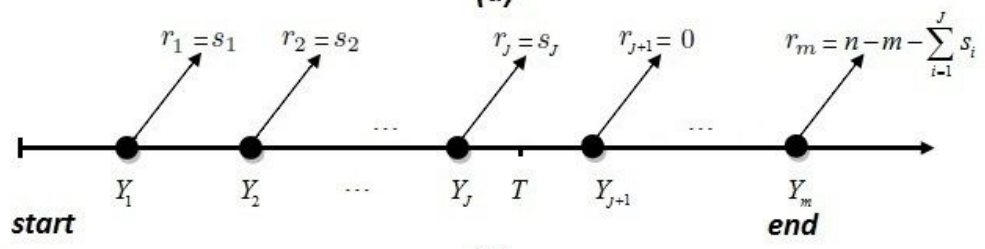

(b)

Figure 2: $\mathrm{Ng}$ et al.'s method.

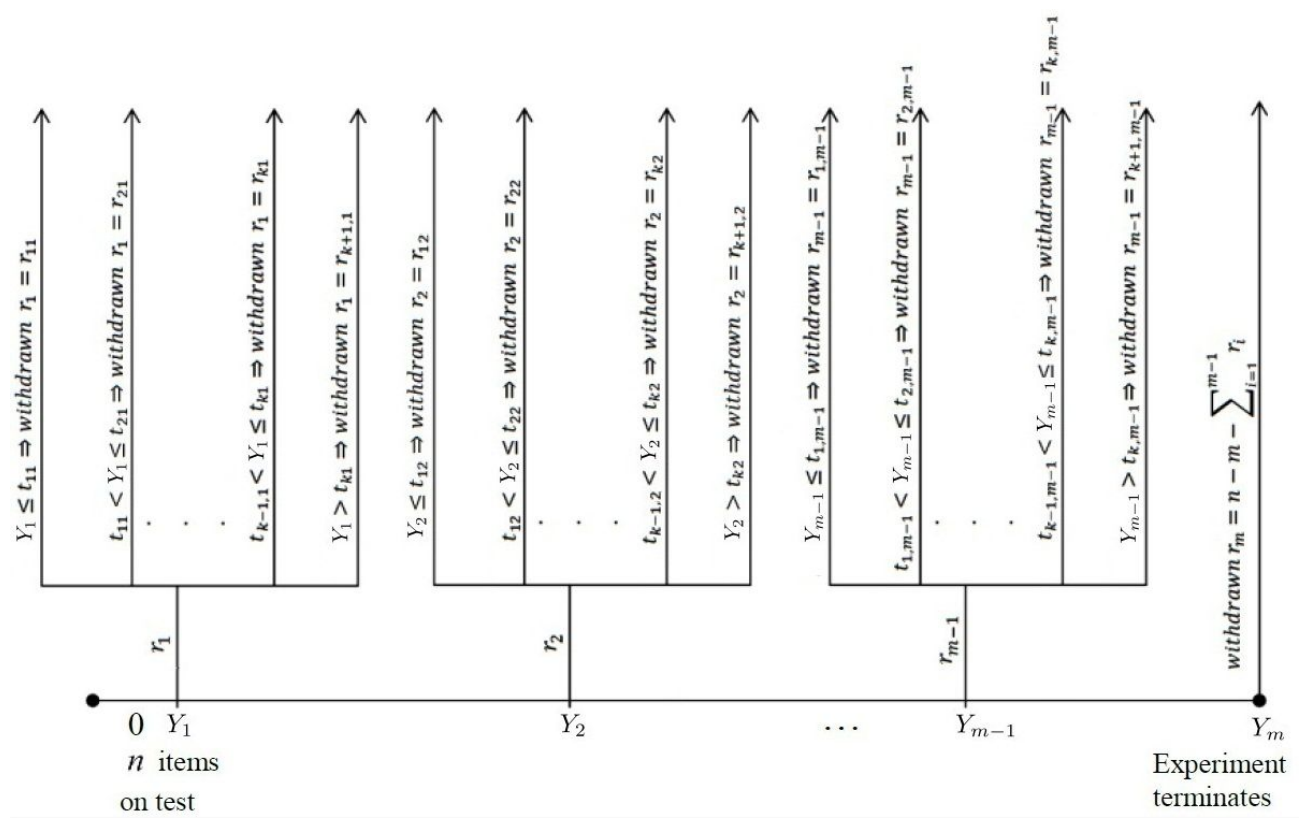

Figure 3: Kinaci’s method.

In advance of the experiment, the experimenter should determine some parameters such as censoring removal schemes, $\boldsymbol{r}_{i}=\left(r_{i, 1}, \ldots, r_{i, m-1}\right), i=1, \ldots, k+1$ provided $r_{i, j} \leq r_{i+1, j}, i=1, \ldots, k$ and $j=1, \ldots, m-1$ and also the vectors of time $\boldsymbol{t}_{i}=\left(t_{i, 1}, \ldots, t_{i, m-1}\right), i=1, \ldots, k$, give, $t_{0, j}=0$ and $t_{k+1, j}=\infty, j=1, \ldots, m-1$. First, all of the items are placed simultaneously on the experiment, then at the first failure time, $R_{1}$ surviving items are immediately removed, where $R_{1}=r_{i, 1}$ if $t_{i-1,1}<Y_{1} \leq t_{i, 1}$, $i=1, \ldots, k+1$. Briefly, at the $j^{\text {th }}$ failure time, $j=1, \ldots, m-1, R_{j}$ surviving items are immediately removed from the experiment, where $R_{j}=r_{i, j}$ if $t_{i-1, j}<Y_{j} \leq t_{i, j}$. Finally, at the $m^{\text {th }}$ failure time all remaining items are removed from the test and the experiment terminates (Figure 3 ). 
The introduced method by Bairamov and Parsi (2011) in a special case of Kinaci's method, because in Kinaci's method if $k=2$ then the Kinaci's method changes to Bairamov's and Parsi's (2011) method.

The Kinaci's scheme is very flexible, but has some disadvantages such that make it difficult to use, for example, the experimenter has to determine $[(2 k+1) *(m-1)]+2$ parameters, also, when the failure rate is low this scheme removes a greater number of items and when the failure rate is not low this scheme removes a less number of items from the test (and vice versa). When researchers use the Type-II progressive censoring method, they may insist on using a special censoring removal scheme because of benefits or limitations (such as time and cost); but in practice, researchers may have some unexpected problems that make them unable to remove the prefixed number of items at the failure time of the items. One of these problems is descending failure rate that makes the experimental duration very long. Let the failure rate be descended, if the prefixed number of units is removed at the failure times, then the failure rate will be further descended and the experimental duration would be very long. One way to overcome this problem is decreasing the number of removals at each step to restrain decreasing failure rate. Now the matter of interest is how many items should be removed such that the researcher observes no decreasing in the failure rate. We need a censoring method that is flexible and requires fewer parameters.

Motivated by this idea, in this study, we modify the Kinaci's method; the modified method has more flexibility and need's fewer parameters, has reasonable manner when the failure rate is low need's fewer parameters. We call this method as "Type-II stepwise progressive censoring" in the next sections. This topic is more explained in the next section.

\section{Type-II stepwise progressive censoring}

Suppose that $n$ independent and identically distributed units are placed on a lifetime experiment. Before the experiment, $\boldsymbol{s}=\left(s_{1}, s_{2}, \ldots, s_{m-1}\right)$ and $\boldsymbol{t}=\left(t_{1}, t_{2}, \ldots, t_{m-1}\right)$ should be determined, where $\boldsymbol{t}$ is a vector of times and $t_{i}$ 's, $i=1, \ldots, m-1$ are ordered. When the experiment runs, at the first failure $\left(y_{1}\right), R_{1}$ surviving units are removed from the experiment where $R_{1}$ is a random variable such that if $t_{1}=0$ then $R_{1}=0$ else is defined as:

$$
R_{1}= \begin{cases}s_{1}, & t_{1}+\frac{y_{1}-t_{1}}{t_{1}} \leq t_{1}, \\ s_{1}-1, & t_{1}<t_{1}+\frac{y_{1}-t_{1}}{t_{1}} \leq t_{1}+\frac{2}{s_{1}\left(s_{1}+1\right)} \\ \vdots & t_{1}+\frac{i(i-1)}{s_{1}\left(s_{1}+1\right)}<t_{1}+\frac{y_{1}-t_{1}}{t_{1}} \leq t_{1}+\frac{i(i+1)}{s_{1}\left(s_{1}+1\right)} \\ s_{1}-i, & t_{1}+\frac{\left(s_{1}-1\right)}{\left(s_{1}+1\right)}<t_{1}+\frac{y_{1}-t_{1}}{t_{1}}\end{cases}
$$

Then the experiment is continued with $n-R_{1}-1$ remaining units. At the $j^{\text {th }}$ failure time $y_{j}$, $2 \leq j \leq m-1, R_{j}$ surviving units are withdrawn from the experiment where if $\left(t_{j}-t_{j-1}\right)>0$, then $R_{j}$ 


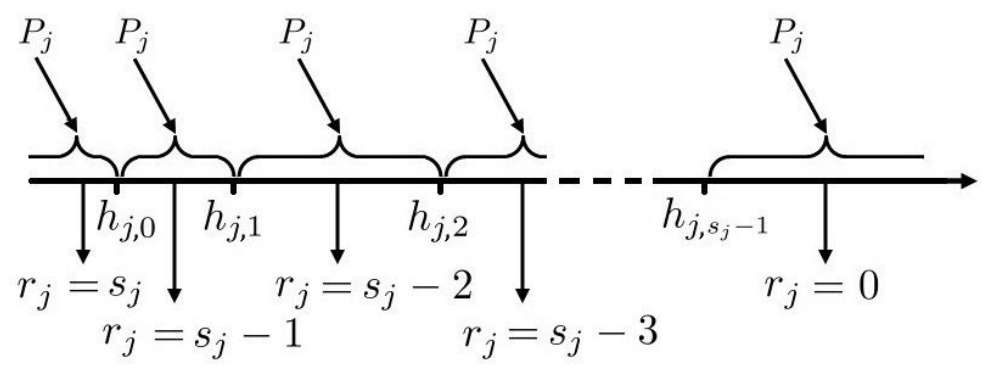

Figure 4: Type-II stepwise progressive censoring.

is a random variable satisfying

$$
R_{j}= \begin{cases}s_{j}, & t_{j}+\frac{y_{j}-t_{j}}{t_{j}-t_{j-1}} \leq t_{j}, \\ s_{j}-1, & t_{j}<t_{j}+\frac{y_{j}-t_{j}}{t_{j}-t_{j-1}} \leq t_{j}+\frac{2}{s_{j}\left(s_{j}+1\right)}, \\ \vdots & t_{j}+\frac{i(i-1)}{s_{j}\left(s_{j}+1\right)}<t_{j}+\frac{y_{j}-t_{j}}{t_{j}-t_{j-1}} \leq t_{j}+\frac{i(i+1)}{s_{j}\left(s_{j}+1\right)}, \\ s_{j}-i, & t_{j}+\frac{\left(s_{j}-1\right)}{\left(s_{j}+1\right)}<t_{j}+\frac{y_{j}-t_{j}}{t_{j}-t_{j-1}}\end{cases}
$$

and if $\left(t_{j}-t_{j-1}\right)=0$ then

$$
R_{j}= \begin{cases}s_{j}, & y_{j} \leq t_{j}, \\ 0, & t_{j}<y_{j} .\end{cases}
$$

Finally, at the $m^{\text {th }}$ failure time, $y_{m}$, all remaining items, $R_{m}$, are removed and the experiment is terminated. We call this method as "Type-II stepwise progressive censoring".

Let $P_{j}=t_{j}+\left(Y_{j}-t_{j}\right) /\left\{\left(t_{j}-t_{j-1}\right) I_{(0, \infty)}\left(t_{j}-t_{j-1}\right)+I_{\{0\}}\left(t_{j}-t_{j-1}\right)\right\}$ and $h_{j, i}=t_{j}+i(i+1) /\left\{s_{j}\left(s_{j}+\right.\right.$ 1) $I_{(0, \infty)}\left(t_{j}-t_{j-1}\right)$, for $j=1, \ldots, m-1$ and $i=0,1, \ldots, s_{j}-1$ where $t_{0}=0$ also $I_{\{b\}}(a)=1$ if $a \in b$ and $I_{\{b\}}(a)=0$, otherwise. By using $P_{j}$ and $h_{j, i}$ notations the definition of $R_{j}$, for $j=1, \ldots, m-1$ and $i=0,1, \ldots, s_{j}-1$ is:

$$
R_{j}= \begin{cases}s_{j}, & -\infty<P_{j} \leq h_{j, 0}, \\ s_{j}-1, & h_{j, 0}<P_{j} \leq h_{j, 1}, \\ \vdots & \\ s_{j}-i, & h_{j, i-1}<P_{j} \leq h_{j, i}, \\ \vdots & \\ 0, & h_{j, s_{j}-1}<P_{j} \leq \infty .\end{cases}
$$

Figure 4 shows the algorithm of the Type-II stepwise progressive censoring at the $j^{\text {th }}$ step, $1 \leq j \leq$ $m-1$. 

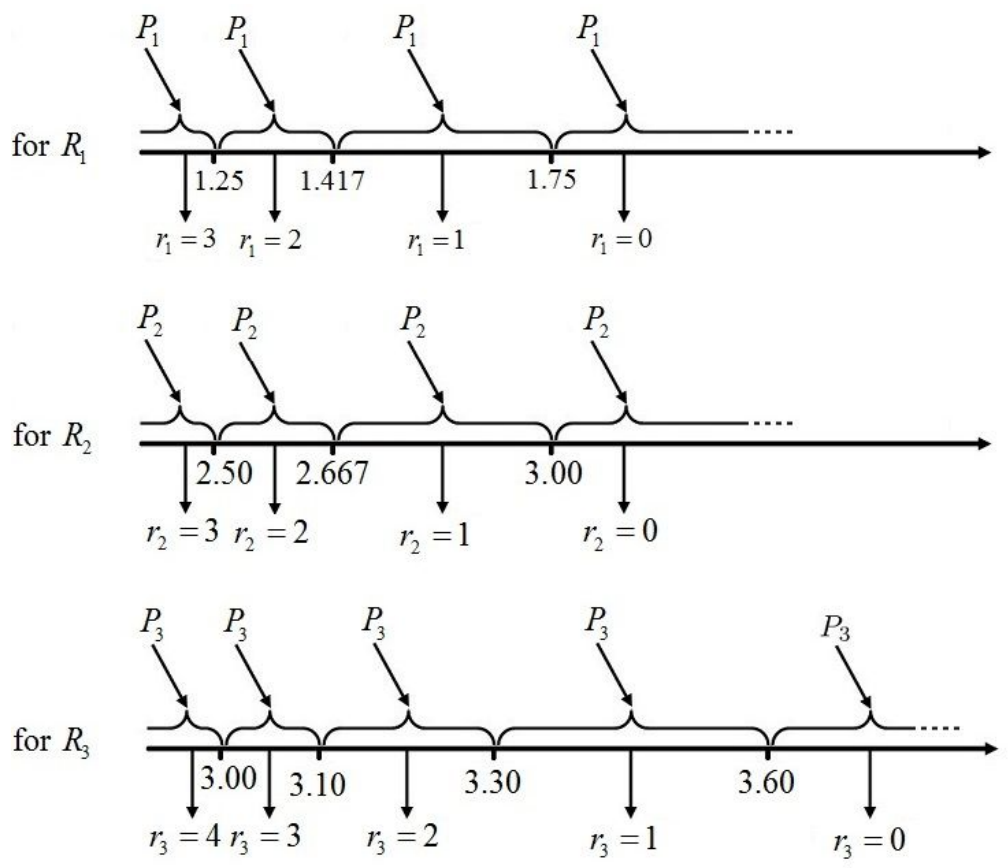

Figure 5: Type-II stepwise progressive censoring for Example 1.

Example 1. Suppose that an experimenter wants to do a lifetime experiment by using the Type-II stepwise progressive censoring method. Moreover, suppose that $n=15, m=4, s_{4}=(3,3,4,1)$ and $\boldsymbol{t}_{3}=(1.25,2.5,3)$. Then, the Figure 5 depicts the number of removals at the all three steps (Example 1). Let $\boldsymbol{y}_{4}=(1.1,2.75,3.1,4)$, then $\boldsymbol{p}_{3}=(1.13,2.7,3.2)$ so, $\boldsymbol{r}_{4}=(3,1,2,5)$.

Henceforth, "Type-II progressive censoring" and "Type-II stepwise progressive censoring" phrases are abbreviated by T2PC and T2SPC, respectively.

\section{The likelihood function of T2SPC and its MLE}

The conditional density function of $R_{j}, 1 \leq j \leq m-1$ can be obtained by:

$$
g_{j}\left(r_{j} \mid y_{j}\right)=\sum_{i=0}^{s_{j}} I_{\left(s_{j}-i\right)}\left(r_{j}\right) I_{\left(h_{j, i-1}, h_{j, i}\right]}\left(p_{j}\right)
$$

and

$$
g_{m}\left(r_{m} \mid y_{m}\right)=I_{n-\sum_{j=1}^{m-1}\left(r_{j}+1\right)}\left(r_{m}\right)
$$

where $h_{j,-1}=-\infty$ and $h_{j, s_{j}}=+\infty$. However, from Cramer and Iliopoulos (2009), the likelihood function of $\mathrm{T} 2 \mathrm{PC}$ is

$$
f_{\mathbf{Y}_{m}, \boldsymbol{R}_{m}}\left(\mathbf{y}_{m}, \boldsymbol{r}_{m}\right)=\prod_{j=1}^{m} f\left(y_{j} \mid \mathbf{y}_{j-1}, \boldsymbol{r}_{j-1}\right) \times \prod_{j=1}^{m} g_{j}\left(r_{j} \mid y_{j}\right)
$$


Hence, the likelihood function of T2SPC is:

$$
f_{\mathbf{Y}_{m}, \boldsymbol{R}_{m}}\left(\mathbf{y}_{m}, \boldsymbol{r}_{m}\right)=C \prod_{j=1}^{m} f\left(y_{j}\right)\left[1-F\left(y_{j}\right)\right]^{r_{j}} \times \prod_{j=1}^{m-1} \sum_{i=1}^{s_{j}-1} I_{\left(s_{j}-i\right)}\left(r_{j}\right) I_{\left(h_{j, i-1}, h_{j, i}\right]}\left(p_{j}\right) \times I_{n-\sum_{j=1}^{m-1}\left(r_{j}+1\right)}\left(r_{m}\right),
$$

where $C$ is a normalizing constant satisfying

$$
\int_{0}^{\infty} \int_{y_{1}}^{\infty} \cdots \int_{y_{m-1}}^{\infty} f_{\mathbf{Y}_{m}, \boldsymbol{R}_{m}}\left(\mathbf{y}_{m}, \boldsymbol{r}_{m}\right)=1
$$

By use of Equation (3.1) the joint likelihood function of $\boldsymbol{Y}=\left(Y_{1}, \ldots, Y_{m}\right)$ and $\boldsymbol{R}=\left(R_{1}, \ldots, R_{m}\right)$ can be written as

$$
L(\mathbf{y}, \mathbf{r} ; \theta)=L_{1}(\mathbf{y} ; \theta \mid \mathbf{r}) \times L_{2}(\mathbf{r} \mid \mathbf{y})
$$

where

$$
L_{1}(\mathbf{y} ; \theta \mid \mathbf{r})=\prod_{j=1}^{m} f\left(y_{j} \mid \mathbf{y}_{j-1}, \boldsymbol{r}_{j-1}\right)
$$

and

$$
L_{2}(\mathbf{r} \mid \mathbf{y})=\prod_{j=1}^{m} g_{j}\left(r_{j} \mid \mathbf{y}_{j}\right)
$$

Since $L_{2}(\mathbf{r} \mid \mathbf{y})$ does not involve in the parameter $\theta$, the maximum likelihood estimators (MLE) of $\theta$ can be obtained by maximizing $L_{1}(\mathbf{y} ; \theta \mid \mathbf{r})$ directly. So, the MLE of the parameter in T2SPC is similar to T2PC.

\section{Marginal distributions}

Let $(\boldsymbol{Y}, \boldsymbol{R})$ denote the T2SPC data from an absolutely continuous population with $\mathrm{CDF} F(\cdot)$ and PDF $f(\cdot)$. This is obviously the marginal distribution of the first T2SPC order statistic is

$$
f_{Y_{1}}(y)=n f(y)[1-F(y)]^{n-1} .
$$

It can be shown that the joint distribution T2SPC order statistics given $\boldsymbol{R}=\boldsymbol{r}$ is similar to the ordinary progressively Type II censored order statistics with censoring removal scheme $\boldsymbol{r}$. Due to the Markovian property of ordinary progressive Type II censored order statistics the marginal distribution of $i^{\text {th }}$ of T2SPC does not depend on $\left(r_{i}, \ldots, r_{m}\right)$. The marginal distribution of $i^{t h}$ ordinary progressive Type II censored order statistics, $Y_{i}, i=2,3, \ldots, m$ is

$$
f_{Y_{i}}\left(y_{i} \mid \mathbf{r}_{i-1}\right)=C_{i-1} f(y) \sum_{k=1}^{m} a_{k, i}[1-F(y)]^{\gamma_{k}-1},
$$

where $C_{i-1}=\prod_{j=1}^{i} \gamma_{j}, a_{k, i}=\prod_{j=k, j \neq k}^{i}\left(1 /\left(\gamma_{j}-\gamma_{k}\right)\right), 1 \leq k \leq i \leq m, m \geq 2, \gamma_{j}=n-\sum_{i=1}^{j-1}\left(r_{i}+1\right)$, $\gamma_{1}=n, n=m+\sum_{j=1}^{m} r_{j}$. For more details, see Kamps and Cramer (2001). 
So, the PDF of $i^{\text {th }}$ T2SPC order statistic can be obtained by

$$
f_{Y_{i}}\left(y_{i}\right)=\sum_{r_{1}=0}^{s_{1}} \cdots \sum_{r_{i-1}=0}^{s_{i-1}} f_{Y_{i}}\left(y_{i} \mid \mathbf{r}_{i-1}\right) P\left(\mathbf{R}_{i-1}=\mathbf{r}_{i-1}\right)
$$

where

$$
P\left(\mathbf{R}_{i}=\mathbf{r}_{i}\right)=\prod_{j=1}^{i} g_{j}\left(r_{j} \mid \mathbf{y}_{j}\right)
$$

See Dey and Dey (2014), Sarhan and Al-Ruzaizaa (2010) and Tse et al. (2000).

We have

$$
\begin{aligned}
g_{j}\left(r_{j} \mid \mathbf{y}_{j}\right)= & I_{\left\{s_{j}-i\right\}}\left(r_{j}\right) P\left(h_{j, i-1}<P_{j} \leq h_{j, i}\right) I_{(0,+\infty)}\left(t_{j}-t_{j-1}\right) \\
& +\left[P\left(-\infty<y_{j} \leq t_{j}\right) I_{s_{j}}\left(r_{j}\right)+P\left(t_{j}<y_{j} \leq+\infty\right) I_{0}\left(r_{j}\right)\right] I_{\{0\}}\left(t_{j}-t_{j-1}\right) \\
= & P\left(t_{j}+\left(t_{j}-t_{j-1}\right) \frac{i(i-1)}{s_{j}\left(s_{j}+1\right)}<y_{j} \leq t_{j}+\left(t_{j}-t_{j-1}\right) \frac{i(i+1)}{s_{j}\left(s_{j}+1\right)}\right) I_{(0,+\infty)}\left(t_{j}-t_{j-1}\right) \\
& +\left[P\left(-\infty<y_{j} \leq t_{j}\right) I_{s_{j}}\left(r_{j}\right)+P\left(t_{j}<y_{j} \leq+\infty\right) I_{0}\left(r_{j}\right)\right] I_{\{0\}}\left(t_{j}-t_{j-1}\right),
\end{aligned}
$$

So

$$
P\left(R_{j}=r_{j} \mid \mathbf{R}_{j-1}=\mathbf{r}_{j-1}\right)=P\left(b_{j, i-1}<y_{j} \leq b_{j, i}\right),
$$

where $b_{j, i}=t_{j}+\left(t_{j}-t_{j-1}\right)\{i(i+1)\} /\left\{s_{j}\left(s_{j}+1\right)\right\}, i=0, \ldots, s_{j}-1, b_{j,-1}=-\infty$ and $b_{j, s_{j}}=+\infty$.

\section{The test duration}

It is often useful to have some information about the test duration time due to the direct relation between the test duration time and cost in practical applications. In ordinary Type II progressive censoring the test termination time is the $m^{\text {th }}$ failure time, so the expected test duration time is equal to the expected $m^{\text {th }}$ failure time. The expected test termination time for the ordinary Type II progressive censoring with $\mathbf{r}=\left(r_{1}, \ldots, r_{m-1}\right)$ is introduced by Balakrishnan and Aggarwala (2000) and is also formulated as

$$
E\left[Y_{m} \mid \mathbf{R}=\mathbf{r}\right]=C(\mathbf{r}) \sum_{l_{1}=0}^{r_{1}} \cdots \sum_{l_{m}=0}^{r_{m}}(-1)^{A} \frac{\left(\begin{array}{l}
r_{1} \\
l_{1}
\end{array}\right) \cdots\left(\begin{array}{l}
l_{m} \\
r_{m}
\end{array}\right)}{\prod_{i=1}^{m} h\left(l_{i}\right)} \int_{0}^{\infty} y f(y) F^{h\left(l_{i}\right)-1}(y) d y,
$$

where $A=\sum_{i=1}^{m} l_{i}, C(\mathbf{r})=\prod_{i=0}^{m-1}\left(n-\sum_{j=1}^{i}\left(r_{j}+1\right)\right), h\left(l_{i}\right)=\sum_{j=1}^{i}\left(l_{j}+1\right)$ and $i$ is the number of failures. For more details, readers can refer to Balakrishnan and Aggarwala (2000), Tse et al. (2000), Sarhan and Al-Ruzaizaa (2010).

Another representation of the expected test duration is also proposed by Kamps and Cramer (2001). This representation is

$$
E\left[Y_{m} \mid \mathbf{R}=\mathbf{r}\right]=C_{i-1} \int_{0}^{\infty} y f(y) \sum_{k=1}^{m} a_{k, i}[1-F(y)]^{\gamma_{k}-1} d y .
$$


Table 1: Data from T2SPC

\begin{tabular}{ccccccc}
\hline \hline$i$ & 1 & 2 & 3 & 4 & 5 & 6 \\
\hline$Y_{i}$ & 0.1788 & 0.2892 & 0.4560 & 0.5184 & 0.8412 & 0.9864 \\
$R_{i}$ & 3 & 3 & 3 & 3 & 1 & 4 \\
\hline \hline
\end{tabular}

Equation (5.2) is obtained directly from Equation (4.2) (Kamps and Cramer, 2001; Bairamov and Parsi, 2011). Due to the simplicity of Equation (5.2), we use the formula to compute the test termination time of T2SPC. In T2SPC, due to random removals, the expected test duration time is defined by

$$
E\left[Y_{m}\right]=E_{\mathbf{R}}\left[E\left[Y_{m} \mid \mathbf{R}\right]\right]
$$

For T2SPC we have

$$
\begin{aligned}
E\left[Y_{m}\right] & =\sum_{r_{1}=0}^{s_{1}} \cdots \sum_{r_{m-1}=0}^{s_{m-1}} P(\mathbf{R}=\mathbf{r}) E_{\mathbf{R}}\left[E\left[Y_{m} \mid \mathbf{R}=\mathbf{r}\right]\right], \\
& =\sum_{r_{1}=0}^{s_{1}} \cdots \sum_{r_{m-1}=0}^{s_{m-1}} P(\mathbf{R}=\mathbf{r}) C_{i-1} \int_{0}^{\infty} y f(y) \sum_{k=1}^{m} a_{k, i}[1-F(y)]^{\gamma_{k}-1} d y .
\end{aligned}
$$

This can be noted that for $i=1, \ldots, m-1$

$$
E\left[R_{i} \mid \mathbf{r}_{i-1}\right]=\sum_{j=0}^{s_{i}} j P\left(R_{i}=j \mid \mathbf{r}_{i-1}\right) .
$$

\section{Real data}

Here there are 23 deep-groove ball bearing failure times. Who first used the data set is Lieblein and Zelen (1956), but some other authors used and discussed about this failure data set. These failure times are

$$
\begin{array}{llllllllll}
0.1788 & 0.2892 & 0.3300 & 0.4152 & 0.4212 & 0.4560 & 0.4848 & 0.5184 & 0.5196 & 0.5412 \\
0.5556 & 0.6780 & 0.6864 & 0.6864 & 0.6888 & 0.8412 & 0.9312 & 0.9864 & 1.0512 & 1.0584 \\
1.2792 & 1.2804 & 1.7340 & & & & & & &
\end{array}
$$

Raqab (2002) shows that the Rayleigh distribution provides a justifiable fit to the ball bearing complete data. Dey and Dey (2014) used this data set to generate progressively Type II censored data using binomial removals. They performed a goodness-of-fit test with a Kolmogorov-Smirnov test and chi square test. They had shown that the Rayleigh distribution has a reasonable fit to the progressively Type II censored ball bearing data. More details about the data set is explained by Caroni (2002). Here we use this data set to generate stepwise progressively Type II censored data. We consider $m=6$, $\boldsymbol{t}=(0.2,0.3,0.5,0.6,0.8)$ and the censoring scheme $\boldsymbol{r}=(3,3,3,3,3,2)$. The observed failure times are given by Table 1 .

Thus, the experiment is continued until 0.9864 and four units are withdrawn at the final censoring step. The total number of progressively censored units is 17 . The effectively applied removal censoring scheme is $\boldsymbol{r}=(3,3,3,3,1,4)$. The lifetimes belonging to the Table 2 are stepwise progressively Type II censored: tics.

Figure 6 depicts the generation procedure of stepwise progressively Type-II censored order statis- 
Table 2: Stepwise progressively Type II censored data

\begin{tabular}{cl}
\hline \hline Censoring time & \multicolumn{1}{c}{ Censored data } \\
\hline 0.1788 & $0.4152,0.6864,0.9312$ \\
0.2892 & $0.3300,0.4212,0.5556$ \\
0.4560 & $0.4848,0.6864,1.734$ \\
0.5184 & $0.5196,0.5412,0.6780$ \\
0.8412 & 0.9312 \\
0.9864 & $1.0512,1.0584,1.2792,1.2804$ \\
\hline \hline
\end{tabular}

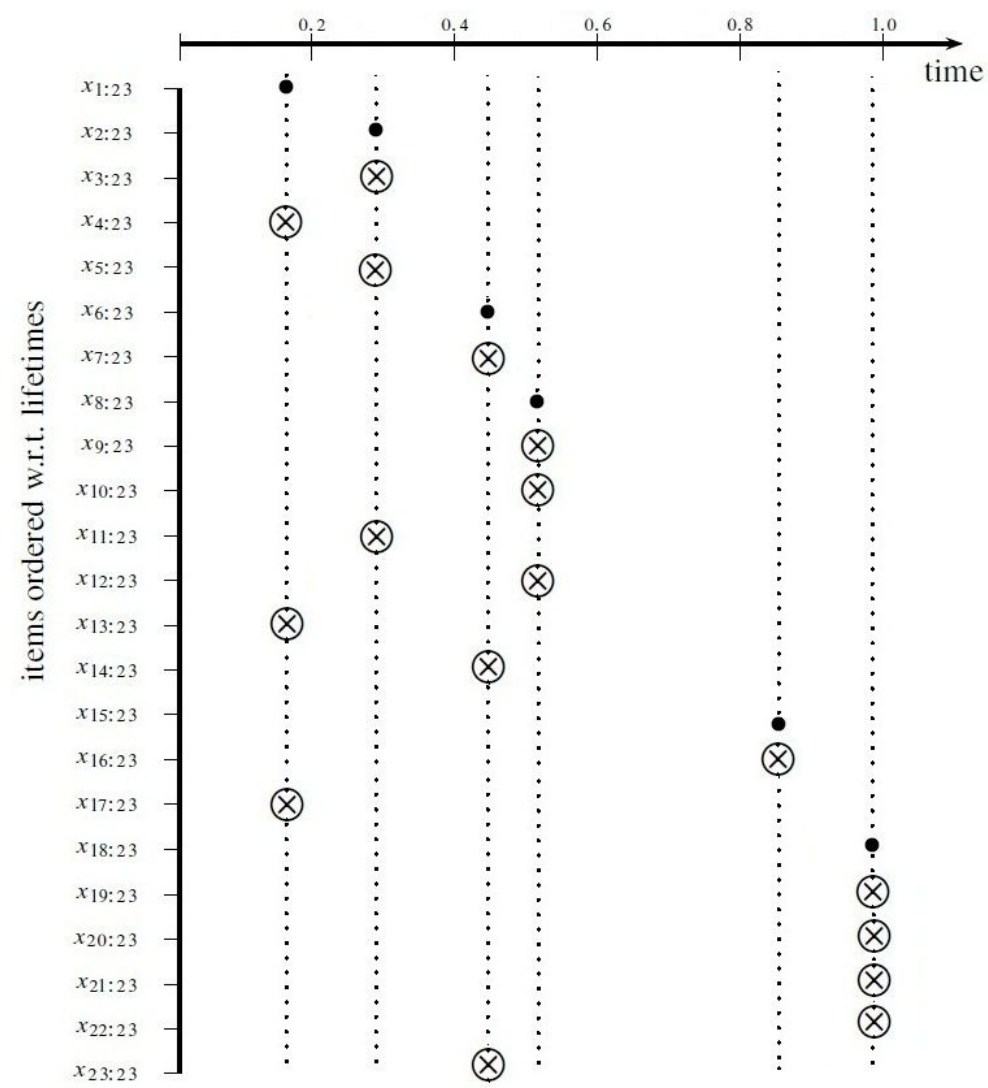

Figure 6: Generation process of a progressively stepwise Type-II censored data set from 23 deep-groove ball bearing failure times (filled circle denotes an observed failure time and open crossed circle denotes a censored value).

\section{Submodels of T2SPC}

In this section, we introduce and discuss about some special cases of T2SPC.

\subsection{T2PC}

First, define $\infty-\infty=0$. If $t_{i}=\infty, i=1,2, \ldots, m-1$, in the time vector $(t)$, then the T2SPC method is similar to the T2PC method, because in this case

$$
R_{j}=s_{j}, \quad j=1,2, \ldots, m-1,
$$


Table 3: The diversity of T2SPC and T2PC. in a same experiment

\begin{tabular}{ccccc}
\hline \hline Censoring Type & Censoring removal scheme & Data & $E\left(X_{m}\right)$ & Estimate \\
\hline T2PC & $5,5,5,5,5$ & $0.13,1.26,1.92,2.38,3.59$ & 3.59 & 0.0898 \\
T2SPC & $5,1,0,5,14$ & $0.13,0.62,1.92,2.12,2.70$ & 2.70 & 0.0875 \\
\hline \hline
\end{tabular}

and Equation (3.2) changes to (7.1).

Also, in this case

$$
f_{\mathbf{Y}_{m}, \boldsymbol{R}_{m}}\left(\mathbf{y}_{m}, \boldsymbol{r}_{m}\right)=C \prod_{j=1}^{m} f\left(y_{j}\right)\left[1-F\left(y_{j}\right)\right]^{r_{j}} \times I_{\left(s_{1}, s_{2}, \ldots, s_{m}\right)}\left(r_{1}, r_{2}, \ldots, r_{m}\right) .
$$

Equation (7.1) is similar to the likelihood function of T2PC by using the censoring removal scheme $s$.

\section{2. $\mathrm{Ng}$ et al.'s generalized T2PC}

In the T2SPC method, if all the $t_{i}$ 's, $i=1,2, \ldots, m-1$ in the time vector is equal to same constant time, namely $T$ then, the T2SPC method is changed to the $\mathrm{Ng}$ et al.'s method and in this case the Equation (3.2) is changed to Equation (7.2).

$$
f_{\mathbf{Y}_{m}, \boldsymbol{R}_{m}}\left(\mathbf{y}_{m}, \boldsymbol{r}_{m}\right)=C \prod_{j=1}^{m} f\left(y_{j}\right)\left[1-F\left(y_{j}\right)\right]^{r_{j}} \times I_{\left(s_{1}, s_{2}, \ldots, s_{J}, 0, \ldots, 0, \sum_{j=J+1}^{m} s_{j}\right)}\left(r_{1}, r_{2}, \ldots, r_{m}\right),
$$

where $J$ is the number of failures that observed before T.

\section{Simulation study for comparison with T2PC}

For illustration the influence of T2SPC against T2PC, first the diversity of these two censors is shown by simulation. In this simulation, the distribution of the simulated data is supposed to be exponential distribution $\operatorname{Exp}(\theta), \theta=0.1$ for $m=5, n=30, t=(0.15,0.2,1.2,2.4)$ and $s=(5,5,5,5,5)$ as censoring removal scheme (Table 3 ).

Here, some comparisons between T2SPC and T2PC by means of simulation are done. The software that is used for the simulation study is R software; with the Monte Carlo method for 10000 times repeats. The simulated data are exponentially distributed $\operatorname{Exp}(\theta), \theta=0.1$. The experiment is simulated for T2SPC and T2PC, respectively. For each case, the empirical maximum likelihood estimator (MLE), the empirical mean of square error (MSE) and the empirical test duration are obtained and are tabulated in Table 4, where

1. $\hat{\theta}_{1}$ is the empirical MLE by use of T2PC.

2. $\hat{\theta}_{2}$ is the empirical MLE by use of T2SPC.

3. $\mathrm{MSE}_{1}$ is the empirical MSE by use of T2PC.

4. $\mathrm{MSE}_{2}$ is the empirical MSE by use of T2SPC.

5. $E\left(X_{m}\right)_{1}$ is the empirical test duration by use of T2PC.

6. $E\left(X_{m}\right)_{2}$ is the empirical test duration by use of T2SPC.

From Tables 4, it is easy to see that $E\left(X_{m}\right)_{2} \leq E\left(X_{m}\right)_{1}$ and $\mathrm{MSE}_{2}$ is equal to $\mathrm{MSE}_{1}$, approximately. From Table 4, for example, for $\mathrm{X}$ having the exponential distribution with parameter $\theta=0.1$ and 
Table 4: Simulations of T2SPC and T2PC

\begin{tabular}{|c|c|c|c|c|c|c|c|}
\hline$n$ & $m$ & \multicolumn{2}{|r|}{$\boldsymbol{r}$} & \multicolumn{4}{|c|}{$t$} \\
\hline 20 & 5 & \multicolumn{2}{|c|}{$3, \ldots, 3$} & & \multicolumn{3}{|c|}{$0.33,0.78,1.45,2.45$} \\
\hline 20 & 10 & \multicolumn{2}{|c|}{$1, \ldots, 1$} & & \multicolumn{3}{|c|}{$0.35,0.77,1.29,1.84,2.5,3.24,4.00,5.64,7.4$} \\
\hline 30 & 5 & \multicolumn{2}{|c|}{$5, \ldots, 5$} & & \multicolumn{3}{|c|}{$0.28,0.59,0.97,1.59$} \\
\hline 30 & 10 & \multicolumn{2}{|c|}{$2, \ldots, 2$} & & \multicolumn{3}{|c|}{$0.24,0.51,0.88,1.26,1.63,2.01,2.75,3.64,4.85$} \\
\hline 50 & 5 & \multicolumn{2}{|c|}{$9, \ldots, 9$} & & \multicolumn{3}{|c|}{$0.17,0.35,0.58,0.94$} \\
\hline 50 & 10 & \multicolumn{2}{|c|}{$4, \ldots, 4$} & & \multicolumn{3}{|c|}{$0.14,0.31,0.51,0.72,0.95,1.22,1.56,2.11,2.70$} \\
\hline 50 & 15 & \multicolumn{3}{|c|}{$10,10,10,0, \ldots, 0,3,2$} & \multicolumn{3}{|c|}{$0.15,0.31,0.58,0, \ldots, 0,6.87$} \\
\hline 70 & 10 & \multicolumn{3}{|c|}{$20,10,10,10,0, \ldots, 0,5,5$} & \multicolumn{3}{|c|}{$0.1,0.24,0.47,0.77,0, \ldots, 0,3.52$} \\
\hline 70 & 20 & \multicolumn{3}{|c|}{$10,10,10,10,0, \ldots, 0,5,5$} & \multicolumn{3}{|c|}{$0.11,0.26,0.44,0.73,0, \ldots, 0,4$} \\
\hline 70 & 30 & \multicolumn{3}{|c|}{$8,8,8,8,0, \ldots, 0,4,4$} & \multicolumn{3}{|c|}{$0.12,0.23,0.37,0.54,0, \ldots, 0,10.64$} \\
\hline 100 & 20 & \multicolumn{3}{|c|}{$16,16,16,16,0, \ldots, 0,8,8$} & \multicolumn{3}{|c|}{$0.067,0.17,0.29,0.45,0, \ldots, 0,5.26$} \\
\hline 100 & 30 & \multicolumn{3}{|c|}{$14,14,14,14,0, \ldots, 0,7,7$} & \multicolumn{3}{|c|}{$0.07,0.15,0.26,0.41,0, \ldots, 0,7.6$} \\
\hline 100 & 50 & \multicolumn{3}{|c|}{$10,10,10,10,0, \ldots, 0,5,5$} & \multicolumn{3}{|c|}{$0.07,0.15,0.25,0.35,0, \ldots, 0,12.65$} \\
\hline$n$ & $m$ & $\hat{\theta}_{1}$ & $\hat{\theta}_{2}$ & $\mathrm{MSE}_{1}$ & $\mathrm{MSE}_{2}$ & $E\left(X_{m}\right)_{1}$ & $E\left(X_{m}\right)_{2}$ \\
\hline 20 & 5 & 0.1244 & 0.1243 & 0.0052 & 0.0051 & 5.7376 & 4.4948 \\
\hline 20 & 10 & 0.1111 & 0.1108 & 0.0015 & 0.0015 & 14.5831 & 11.5472 \\
\hline 30 & 5 & 0.1252 & 0.1250 & 0.0056 & 0.0051 & 3.8178 & 2.9600 \\
\hline 30 & 10 & 0.1112 & 0.1112 & 0.0016 & 0.0015 & 9.7833 & 7.4881 \\
\hline 50 & 5 & 0.1252 & 0.1252 & 0.0052 & 0.0052 & 2.2939 & 1.7631 \\
\hline 50 & 10 & 0.1109 & 0.1107 & 0.0015 & 0.0015 & 5.8841 & 4.2952 \\
\hline 50 & 15 & 0.1075 & 0.1068 & 0.0008 & 0.0008 & 13.9733 & 12.5116 \\
\hline 70 & 10 & 0.1108 & 0.1104 & 0.0014 & 0.0014 & 5.4889 & 5.2154 \\
\hline 70 & 20 & 0.1070 & 0.1064 & 0.0007 & 0.0006 & 10.7499 & 10.5180 \\
\hline 70 & 30 & 0.1037 & 0.1033 & 0.0003 & 0.0003 & 15.6449 & 14.9490 \\
\hline 100 & 20 & 0.1054 & 0.1053 & 0.0006 & 0.0005 & 7.8518 & 7.4344 \\
\hline 100 & 30 & 0.1039 & 0.1036 & 0.0003 & 0.0003 & 11.3327 & 10.8657 \\
\hline 100 & 50 & 0.1019 & 0.1018 & 0.0002 & 0.0002 & 18.0847 & 17.5411 \\
\hline
\end{tabular}

$n=20, m=5$, if we choose the censoring removal scheme as $\boldsymbol{r}=(3,3,3,3)$ and the vector of times as $t=(0.33,0.78,1.45,2.45)$, then the empirical MLE, the empirical MSE and the empirical test duration for T2PC will be $0.1244,0.0052$ and 5.7376, respectively. In the case of the T2SPC method the empirical MLE, the empirical MSE and the empirical test duration will be $0.1243,0.0051$ and 4.4948, respectively. Thus, the T2SPC method decreases the test duration without reducing the deduction quality. These results provide important advantages to life test experimenters.

\section{Conclusions}

In this paper, a new flexible generalized Type II progressive censoring was introduced and was named by "Type-II stepwise progressive censoring". Table 3 indicates the results of a comparison by use of simulation. Table 3 also shows the estimation of the parameter using the T2SPC method was as well as the estimation of parameter using T2PC. Moreover, the failure rate increases because the termination time of the experiment decreases by use of the new method. Whenever an experimenter wants to use the T2PC method, the T2SPC method appears a more flexible and accurate method because if the failure rate is not low practically the censoring removal scheme that is used in the experiment is similar to the censoring removal scheme in T2PC but if the failure rate is low, this censoring method removes some less than the predetermined censoring removal scheme. We can say the introduced method can control the test duration. Moreover the number of the required parameters in T2SPC and Kinaci's method is $2 m-1$ and $[(2 k+1)(m-1)]+2$, respectively. Furthermore, in Kinaci's method by use of $k$ various censoring removal schemes the number of possible resulting censoring removal 
scheme is $k^{m}$,whereas in the new introduced method by use of one censoring removal scheme the number of possible censoring removal scheme is equal to $\prod_{i=1}^{m}\left[\left(s_{i}+1\right)\right.$ !]. Using the T2SPC instead T2PC method is therefore completely justifiable and reasonable.

\section{Acknowledgement}

The authors would like to thank the CSAM and AE and anonymous referees for their constructive comments and suggestions which have improved the manuscript.

\section{References}

Bairamov I and Parsi S (2011). On flexible progressive censoring, Journal of Computational and Applied Mathematics, 235, 4537-4544.

Balakrishnan N (2007). Progressive censoring methodology: an appraisal (with discussions), Test, 16, 211-296.

Balakrishnan N and Aggarwala R (2000). Progressive Censoring: Theory, Methods, and Applications, Birkhäuser, Boston.

Balakrishnan N, Burkschat M, Cramer E, and Hofmann G (2008). Fisher information based progressive censoring plans, Computational Statistics and Data Analysis, 53, 366-380.

Balakrishnan N and Cramer E (2014). The Art of Progressive Censoring, Springer, New York.

Balakrishnan N, Cramer E, and Iliopoulos G (2014). On the method of pivoting the CDF for exact confidence intervals with illustration for exponential mean under life-test with time constraints, Statistics and Probability Letters, 89, 124-130.

Burkschat M (2008). On optimality of extremal schemes in progressive Type-II censoring, Journal of Statistical Planning and Inference, 138, 1647-1659.

Burkschat M, Cramer E, and Kamps U (2006). On optimal schemes in progressive censoring, Statistics and Probability Letters, 76, 1032-1036.

Caroni C (2002). The correct ball bearings data, Lifetime Data Anal, 8, 395-399.

Cohen AC (1963). Progressively censored samples in life testing, Technometrics, 5, 327-329.

Cramer E (2014). Extreme value analysis for progressively Type-II censored order statistics, Communications in Statistics-Theory and Methods, 43, 2135-2155.

Cramer E and Iliopoulos G (2009). Adaptive progressive Type-II censoring, Test, 19, 342-358.

Cramer E and Kamps U (2001). Estimation with sequential order statistics from exponential distributions, Annals of the Institute of Statistical Mathematics, 53, 307-324.

Dey S and Dey T (2014). Statistical inference for the Rayleigh distribution under progressively TypeII censoring with binomial removal, Applied Mathematical Modelling, 38, 974-982.

Ghitany ME, Al-Jarallah RA, and Balakrishnan N (2013). On the existence and uniqueness of the MLEs of the parameters of a general class of exponentiated distributions, Statistics, 47, 605612.

Ghitany ME, Tuan VK, and Balakrishnan N (2014). Likelihood estimation for a general class of inverse exponentiated distributions based on complete and progressively censored data, Journal of Statistical Computation and Simulation, 84, 96-106.

Herd RG (1956). Estimation of parameters of a population from a multi-Censored Sample, Phd Thesis, Iowa State College, Ames, Iowa.

Kamps U and Cramer E (2001). On distributions of generalized order statistics, Statistics, 35, 269280.

Kang SB and Seo JI (2011). Estimation in an exponentiated half logistic distribution under progres- 
sively Type-II censoring, Communications for Statistical Applications and Methods, 18, 657366.

Kinaci I (2013). A generalization of flexible progressive censoring, Pakistan Journal of Statistics, 29, $377-387$.

Krishna H and Kumar K (2013). Reliability estimation in generalized inverted exponential distribution with progressively Type II censored sample, Journal of Statistical Computation and Simulation, 83, 1007-1019.

Lieblein J and Zelen M (1956). Statistical investigation of the fatigue life of deep-groove ball bearings, Journal of Research of the National Bureau of Standards, 57, 273-316.

$\mathrm{Ng}$ HKT, Kundu D, and Chan PS (2009). Statistical of analysis of exponential lifetimes under an adaptive Type-II progressive censoring scheme, Naval Research logistics, 56, 687-698.

Pakyari R and Balakrishnan N (2013). Goodness-of-fit tests for progressively Type-II censored data from location-scale distributions, Journal of Statistical Computation and Simulation, 83, 167178.

Raqab MZ (2002). Inference for generalized exponential distribution based on record statistics, Journal of Statistical Planning and Inference, 104, 339-350.

Rezapour M, Alamatsaz MH, and Balakrishnan N (2013a). On properties of dependent progressively Type-II censored order statistics, Metrika, 76, 909-917.

Rezapour M, Alamatsaz MH, Balakrishnan N, and Cramer E (2013b). On properties of progressively Type-II censored order statistics arising from dependent and nonidentical random variables, Statistical Methodology, 10, 58-71.

Sarhan AM and Al-Ruzaizaa A (2010). Statistical inference in connection with the Weibull model using Type-II progressively censored data with random scheme, Pakistan Journal of Statistics, 26, 267-279.

Seo JI and Kang SB (2014). Predictions for progressively Type-II censored failure times from the half triangle distribution, Communications for Statistical Applications and Methods, 21, 93-103.

Tse SK, Yang C, and Yuen HK (2000). Statistical analysis of Weibull distributed lifetime data under Type II progressive censoring with binomial removals, Journal of Applied Statistics, 27, 10331043. 